\title{
Un método de mejora de proceso para pymes en países en desarrollo
}

\section{INTRODUCCIÓN}

Las pequeñas y medianas empresas (pymes) y las microempresas son un importante sector en la economía de los países en desarrollo; por ello, resulta prioritario que estas empresas logren un nivel competitivo de clase mundial. Para lograr esto se requiere que utilicen técnicas y herramientas de gestión adecuadas, es decir, eficaces, eficientes, sencillas y que no impliquen altos costos para facilitar la ejecución de proyectos de desarrollo, promoviendo el espíritu competitivo en sus directores y accionistas.

Precisamente, la investigación sobre la que se basa este artículo tuvo como objetivo principal encontrar formas adecuadas para el mejoramiento de las pymes en el Perú y hacer de ellas empresas competitivas en el ámbito internacional. En otras palabras, se trata de estudiar los pasos que deben dar las pymes para su mejora, partiendo de su realidad y de sus propias necesidades, en especial de las referidas a recursos humanos, sus capacidades y calificaciones, así como a la restringida capacidad de inversión que poseen y a la necesidad de contar con un modelo de mejoramiento que sea efectivo, a pesar de sus limitaciones. Se debe tener en cuenta incluso la desventaja que significa para el empresario manejar su negocio con sus familiares más cercanos, con deficiencias en el entrenamiento y las capacidades administrativas, como ocurre con frecuencia en nuestro país, donde se 
toman decisiones en áreas en las que, por lo general, solo actúan profesionales especializados (Mendes y Lourenço).

Para efectos del trabajo se tomaron dos acciones principales:

- Elaborar un método de mejoramiento sencillo, de bajo costo y práctico para las pymes, utilizando técnicas y herramientas de gestión simples y efectivas.

- Comprobar el método, verificar la factibilidad de aplicación del modelo y asegurar la obtención de resultados favorables.

\section{LAS PYMES EN EL PERÚ}

El Perú cuenta con más de 27 millones de habitantes, según el último cernso nacional, una población económicamente activa (PEA) de 11,4 millones de personas, un Producto Bruto Interno (PBI) de US\$54,25.000 millones y un PBI per cápita de US\$2.170 dólares ( $E l$ Comercio, 2001: 6-7). Algunas investigaciones estiman que en el 2025 la población habrá crecido en 13,05 millones de habitantes, es decir, un 46,69\%, lo que afectará el mercado laboral que difícilmente podrá absorber a toda la población potencialmente activa y, en consecuencia, exigirá demasiado a los sistemas sociales, produciendo un mayor número de trabajadores no calificados (Wohlcke, 2001: 8-20).

A estas cifras se agregan las siguientes: el 99,6\% de la fuerza laboral del país está concentrado en la microempresa y la pequeña empresa (Infante, 2002: b2) el 98\% del total de unidades económicas se concentra en este sector, donde el $92 \%$ corresponde a pymes con menos de cinco trabajadores, que generan el $75 \%$ de empleos y contribuyen con el 47\% del PBI (García, 2000: 50-52).

En el Perú se consideran microempresas a las unidades económicas registradas legalmente que venden menos de S/.144.000 (US\$41.146) anuales; pequeñas empresas son las que registran ventas anuales mayores de S/.144.000 y menores de US\$750.000; y medianas empresas las que venden más de US\$750.000. En 1996 se estimó que existían en el Perú más de 450.000 microempresas, alrededor de 100.000 pequeñas empresas y 4.593 medianas empresas (Caja pymes).

No hay parámetros que definan las pymes de manera uniforme en todos los países, pero es innegable la importancia que han alcanzado 
tanto en los países desarrollados como en los que se encuentran en camino al desarrollo. En el Brasil se estima que más de 70 millones de personas, de un total de 170 millones de habitantes, dependen directamente de las actividades de las pymes formales e informales, y que entre el $2 \%$ y $3 \%$ de los US $\$ 55.000$ millones que exportaron en el 2001 provinieron de las pymes (Dellaméa). En Taiwan, en 1997 existían 1.020.435 pymes, el 97,81\% de las empresas taiwanesas, que daban trabajo a 7.197 .000 personas, el $79,43 \%$ de la población activa (Government Information Office, República de China).

Pero la magnitud de su presencia sirve fundamentalmente para expresar la situación técnica, económica y social de los países (Valdivia, 2002: b2). Se estima que en el 2002 más de 250 pymes del sector confecciones del Perú han tenido en conjunto un ingreso de US\$15.000.000 anuales, mientras que una sola gran empresa confeccionista peruana exportó esa misma cifra en un cuatrimestre (Valdivia, 2002: b2). En el Brasil, las pymes representan el 13\% de las empresas que exportan, sin embargo, el valor de sus exportaciones asciende al 2\%-3\% del total del valor exportado (Dellaméa, ). En Taiwan, donde representan el $97,81 \%$ de las empresas, vendieron por un valor de US $\$ 209.000$ millones, solo el 32,11\% de las ventas totales, y exportaron por un valor de US\$59,500 millones, solo el 48,77\% de las exportaciones (Government Information Office, República de China).

Para que las pymes logren la competitividad internacional y, por consiguiente, el desarrollo a mediano y largo plazos, de modo que desempeñen el papel protagónico que se les asigna como creadoras de puestos de trabajo y de riqueza, se deberán formular políticas y medidas como la creación de un ambiente propicio para su competitividad, el fomento de la cooperación entre las empresas, la promoción del crecimiento de las empresas, el establecimiento de un fondo para el desarrollo, el establecimiento de servicios que les permitan mejorar la educación y el nivel tecnológico, especialmente en lo relacionado con la investigación y la producción, sin dejar de lado la mercadotecnia y la administración.

En la actualidad, la liberalización y globalización de los mercados exigen nuevos conocimientos y capacidades para lograr el delicado equilibrio entre los correspondientes pares de valores que especifican maneras de actuar, conductas o acciones positivas contrarias, como la adaptabilidad-continuidad que requieren las empresas en un mercado globalizado y que podrían resultar en desorientación y rigidez si no 
existe la capacidad gerencial para negociar una solución equilibrada de compromiso, pues una exagerada adaptabilidad puede terminar en la desorientación del personal, así como una extremada continuidad puede causar la rigidez de la empresa (Greis, 2001).

\section{CARACTERÍSTICAS DE LAS PYMES EN EL PERÚ}

Algunas características de las pymes en el Perú se aprecian en los conceptos y comentarios recogidos por los estudiosos del tema, que han sido publicados en libros y en una diversidad de medios. Hay varios tipos de pymes y microempresas en el Perú; un grupo importante de ellas tiene bajos ingresos, son informales y está administrado por personas con experiencia en el rubro, pero con escasa formación técnica y en gestión empresarial. Otro grupo tiene mejores ingresos y resultados debido a una mejor preparación del personal directivo. Por último, un tercer grupo - menos del 5\% del sector de las pyme- tiene buenos ingresos y resultados gracias a una mejor tecnología y a que son dirigidas por personas que cuentan con formación profesional (Valdivia, 2002). Téngase en cuenta que casi el 38\% de los empresarios de las pymes peruanas no tienen instrucción o solo tienen educación primaria, y que únicamente el 17\% de ellos tiene educación superior (García, 2000: 50-52).

Las pymes del Perú tienen características similares a las de otros países en desarrollo, pero presentan ciertas peculiaridades como consecuencia del entorno y de las circunstancias que vive el país.

Las principales ventajas de estas pymes son su flexibilidad para incorporar técnicas e innovaciones; el nivel de compromiso del empresario que invierte su patrimonio y lo somete a los resultados de su gestión empresarial; la atención personalizada a clientes y proveedores; y el apoyo que reciben de instituciones y entidades internacionales y nacionales, públicas y privadas (Del Carpio, 2000: 53-55). Este apoyo va desde la donación para impulsar su competitividad y desarrollo hasta las compras del Estado, que en el 2002 presentó más de 600 planes anuales de adquisiciones dirigidos a favorecer a las pymes y microempresas peruanas (Infante, 2002: b2).

Algunas desventajas de las pymes y microempresas del Perú son la falta de articulación con la mediana y gran empresa; las dificultades para conformar alianzas; la deficiente cultura productiva; el bajo nivel 
tecnológico; el deficiente manejo contable-económico-financiero; la falta de capacidad para exportar; el escaso desarrollo empresarial; y la baja competitividad debido a deficiencias en la calidad, productividad y servicio (García, 2000: 50-52). A esto se une la problemática que se aprecia cuando se analiza la competitividad desde su enfoque sistémico (Meyer-Stamer, 1997: 8-12), y se observan las deficiencias y carencias que, en lo metaeconómico, dificultan la existencia de un enfoque de desarrollo, una capacidad estratégica y una actitud favorable al cambio, capaz de crear una cultura industrial; asimismo, en lo macroeconómico impiden la existencia de una condición marco estable, indispensable para formular estrategias a largo plazo; finalmente, en el aspecto mesoeconómico no fomentan el desarrollo de sectores o áreas mediante políticas específicas de localización que permitan fortalecer un sector o una región. Para lograr la competitividad internacional no es suficiente - aunque es imprescindible - desarrollar y asimilar nuevas tecnologías y hacer uso de la capacidad de innovación en el campo macroeconómico (Sangmeister y Fuentes, 2002: 8-12). Se requiere la aplicación de una moderna política de desarrollo formulada por los agentes relevantes - Estado, empresas y asociaciones gremiales-debidamente articulados.

Estas características sugieren una solución que comprenda la aplicación de conceptos y herramientas relativos a los aspectos técnicos y de gestión, y el empleo de la consultoría empresarial como agente de cambio. Esto se podría lograr también mediante la preparación de consultores internos, tratando de aprovechar mejor los recursos y facilitando la aplicación en forma autónoma y rentable de los conceptos y herramientas proporcionadas por la consultoría.

\section{EL CONCEPTO DE PYME Y SU DESARROLLO}

Se debe partir del hecho de que las pymes tienen una organización estructural y una cultura administrativa que difiere de aquellas de las empresas grandes; por ello, no se pueden aplicar directamente las experiencias desarrolladas en las empresas grandes (Rantanen, Tenhunen y Rehhn). Además, se debe tener presente el proceso normal para el cambio o mejora, que incluye un análisis que permite llegar a conclusiones que pueden servir como base para elaborar una propuesta de recomendaciones tendientes a conseguir la mejora. 
También se debe tener en cuenta que las características de las pymes aconsejan utilizar métodos sencillos, fáciles de comprender, rápidos de aprender y factibles de aplicar en forma autónoma.

El modelo de un proceso de consultoría que posibilite el mejoramiento de una empresa es presentado de manera similar por diferentes autores. Algunos dividen el proceso en las etapas de captación de clientes, elaboración de oferta y estipulación del contrato, planificación del diagnóstico, análisis de la problemática, alternativas de solución, planeamiento del desarrollo, presentación de la propuesta, ejecución de la propuesta, elaboración del informe final y seguimiento (Barcelli, 2003). Otros dividen estas fases en: contacto con el cliente, captación de clientes, elaboración de la oferta, estructura del contrato, desarrollo de la consultoría y seguimiento (Niedereichholz, 1998). Por último, el manual de consultoría de la OIT divide el proceso en: iniciación, diagnóstico, planificación de medidas, aplicación y terminación (OIT, 1997: 24-29).

Sin dejar de reconocer la importancia de todas las fases o etapas de una consultoría, al enfocar el desarrollo del concepto hay dos temas fundamentales que son considerados por todos los autores: el primero, el diagnóstico de la empresa, que mediante procedimientos analíticos permite entender a la empresa en su conjunto, explicar su situación, señalar los sectores y procesos que impiden o limitan su desarrollo e indicar en forma específica las principales deficiencias encontradas, y el segundo, el planeamiento y desarrollo de alternativas de solución de los problemas, donde empleando técnicas o herramientas de la ingeniería y productos o instrumentos propios de la consultoría, se buscan las alternativas de solución y se planea la aplicación de la elegida para el mejoramiento de la empresa.

Por lo tanto, el desarrollo de un método para el mejoramiento de las pymes tendrá que incluir el desarrollo, la identificación, la selección y la implementación de técnicas y herramientas sobre los siguientes aspectos:

- Análisis de la empresa: Para definir cuáles son los sectores que presentan deficiencias, qué funciones o procesos son los más débiles y, por lo tanto, limitan a la empresa. Se consideraron cinco métodos de diagnóstico para realizar el estudio: análisis estratégico, medición de la productividad, análisis factorial, análisis técnico-económico-financiero y análisis funcional (Barcelli, 2002). Después de 
considerable reflexión, discusión y deliberación se eligió el método de diagnóstico del análisis factorial.

- Análisis de los procesos: Para estudiar en detalle las actividades y tareas que convierten la corriente de ingresos al proceso en una corriente de salida dirigida hacia el cliente -interno o externo- y señalar en forma precisa dónde se deberán tomar medidas que permitan corregir las deficiencias. No obstante su sencillez, se estimó insuficiente el empleo del simple "diagrama de procesos" (flowchart), debido a su limitación en lo que se refiere al nivel de detalle (Barber), por lo tanto se consideraron dos métodos de análisis de procesos: el análisis de la necesidad de informaciones mediante la elaboración de planes de flujo de informaciones y el registro de datos de una empresa (Duisberg, 1995: 43-49), y el análisis del flujo de valor mediante la utilización de las siete herramientas de mapeo del flujo de valor (Hines). Después de reflexionar, discutir y deliberar se eligió el empleo de un plan del flujo de informaciones y de una matriz de respuesta de la cadena de suministros o mapeo de procesos basado en el tiempo.

- Planteamiento de acciones para la mejora: Para elaborar un plan que facilite el mejoramiento de los procesos, las actividades y tareas, y por ende la mejora de la empresa, se consideraron los siguientes enfoques: las herramientas y técnicas de la calidad que incluyen el Círculo de Deming y el Método de los Siete Pasos, además de varias técnicas estadísticas: la fijación de metas; el desarrollo de los productos de consultoría; el análisis de la complejidad y la optimización de procesos empresariales; la evaluación de alternativas de solución; el análisis del valor de utilidad y análisis de riesgos, y el planeamiento del desarrollo (Niedereichholz, 1998); y el Kaizen, que incluye el despliegue de políticas y objetivos, el sistema de sugerencias, los pequeños grupos de trabajo, la gestión con calidad total, el mantenimiento productivo total, y el "just in time" (Masaaki, 1998: 7-10). Después de una considerable reflexión, discusión y deliberación se eligió el Kaizen.

\section{DISEÑO DEL MÉTODO DE MEJORAMIENTO}

El modelo de mejoramiento se diseñó considerando las técnicas y herramientas seleccionadas para cada una de las etapas: el análisis de la empresa, el análisis de los procesos y la planificación de las mejo- 
ras. Las técnicas y herramientas fueron seleccionadas contemplando varios aspectos; en primer lugar, que sean sencillas de enseñar y aprender; en segundo término, que se puedan aplicar fácilmente, y por último, que sean efectivas.

\subsection{Análisis factorial}

Este método de diagnóstico se fundamenta en la identificación de los factores incidentales en el desempeño técnico, económico y financiero de la empresa y de sus elementos constitutivos, cuya calificación permitirá estimar la efectividad de cada factor y, por ende, determinar cuáles son los factores cuyas deficiencias están limitando el desempeño de la empresa.

El método considera los siguientes pasos:

- Se definen los factores de operación más significativos para la empresa, aquellos que inciden en el desarrollo de sus actividades.

- Se señalan los elementos característicos más importantes de cada factor, que al ser evaluados permitirán deducir el nivel de efectividad del factor.

- Se debe obtener información del desempeño y situación de cada elemento, dándole el calificativo correspondiente.

- Con la calificación de cada elemento, se calcula la efectividad de cada factor.

Se prepara un gráfico comparativo que permita visualizar cuáles son los factores más débiles y que, por lo tanto, están limitando a la empresa.

De esta manera se logran determinar los sectores empresariales que requieren atención y se puede decidir acerca de las medidas que se deben tomar y sus prioridades. 


\section{Análisis de la empresa mediante un diagnóstico según el método del análisis factorial}

\begin{tabular}{|c|c|c|c|c|}
\hline Centros de actividades: & A & B & C & \\
\hline Objetivo: & Objetivo principal & Objetivo principal & Objetivo principal & \\
\hline Factores críticos: & & & & Conclusiones \\
\hline \multicolumn{5}{|l|}{ Producción } \\
\hline \multicolumn{5}{|l|}{ Personal } \\
\hline \multicolumn{5}{|l|}{ Competencias } \\
\hline \multicolumn{5}{|l|}{ Costos } \\
\hline \multicolumn{5}{|l|}{ Objetivos } \\
\hline \multicolumn{5}{|l|}{ Motivación } \\
\hline \multicolumn{5}{|l|}{ Control } \\
\hline \multicolumn{5}{|l|}{ Programas } \\
\hline \multicolumn{5}{|l|}{ Métodos } \\
\hline \multicolumn{5}{|l|}{ Información } \\
\hline \multicolumn{5}{|l|}{ Maquinaria } \\
\hline \multicolumn{5}{|l|}{ Ambiente } \\
\hline \multicolumn{5}{|l|}{ Resultados } \\
\hline Conclusiones: & & & & \\
\hline
\end{tabular}

\subsection{Plan de flujo de informaciones}

Partiendo de la importancia de la información como elemento de la organización empresarial y sabiendo que nada sucede si no existe una información adecuada, se requiere visualizar, específicamente, el flujo de las informaciones en los sectores deficientes de la empresa, de modo que se pueda observar y apreciar si el flujo de la información es el adecuado, considerando emisor, medio de información, receptor, cantidad, contenido y oportunidad.

El plan de flujo de informaciones de un sector de la empresa se elabora mostrando en un dibujo todos los puestos de trabajo del sector y todas las comunicaciones entre ellos, señalando informante, medio de transmisión y destinatario de cada información, para lo cual es conveniente establecer en forma previa una simbología sencilla para la representación gráfica.

De esta forma se logran visualizar las deficiencias de la información, las carencias y los excesos de información; se pone de manifiesto si el trámite documentario cumple con su cometido y si el sistema de información es eficiente y garantiza que todos los puestos de trabajo 
reciban las informaciones necesarias para su correcto desempeño, proporcionando la información requerida, en el momento oportuno, en el lugar correcto y con los medios adecuados.

\section{Análisis de los procesos utilizando un plan de flujo de informaciones}

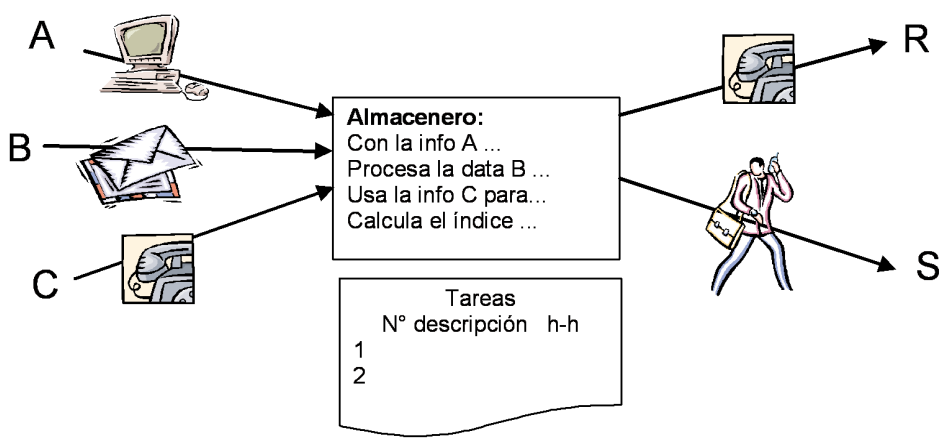

Plan de flujo de informaciones actual

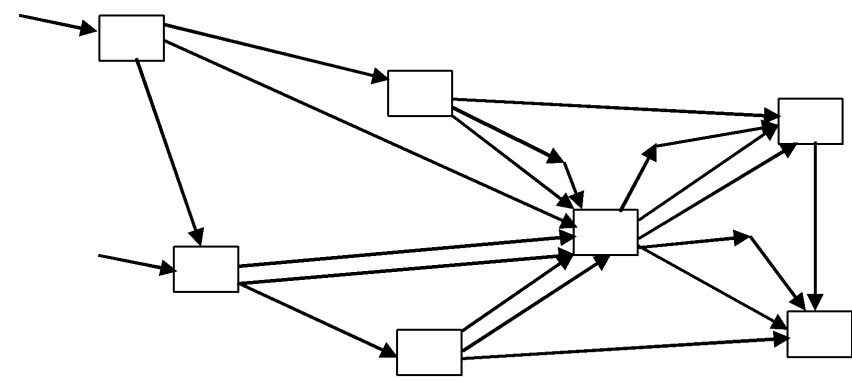

Plan de flujo de informaciones propuesto

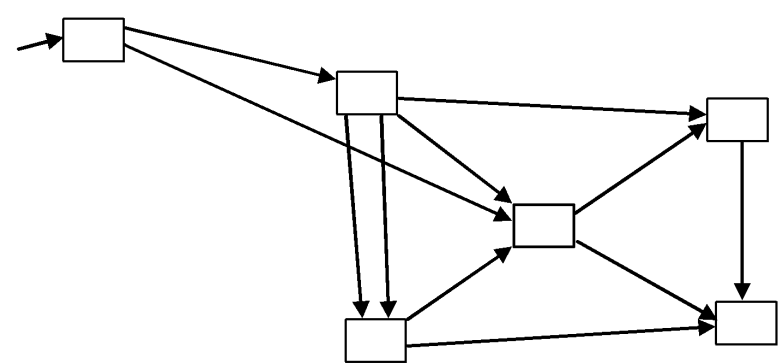




\subsection{MAPEO DE PROCESOS BASADO EN EL TIEMPO}

Este método de análisis del flujo de valor es fundamental para detectar las esperas o demoras y los inventarios innecesarios, dos de los más usuales despilfarros que se encuentran en las empresas. Este mapeo permite visualizar en detalle los procesos y el desempeño de cada una de las operaciones.

El mapeo de procesos basado en el tiempo o matriz de respuesta de la cadena de suministros se realiza en un sencillo diagrama ortogonal, donde se muestra, en el eje horizontal, el tiempo de ejecución (lead time) de cada operación del proceso de un producto, bien o servicio; y en el eje vertical, el equivalente en tiempo de la cantidad promedio de inventarios existentes o de servicios pendientes de atender antes de cada operación. Sumando ambos totales se tendrá el tiempo total de respuesta del proceso.

Este mapeo de procesos permite analizar tanto los tiempos de ejecución (lead time), como los tiempos acumulados por trabajos pendientes en los procesos, de modo que se puedan tomar medidas para mejorarlos.

\section{Análisis de los procesos utilizando un mapeo de procesos basado en el tiempo}

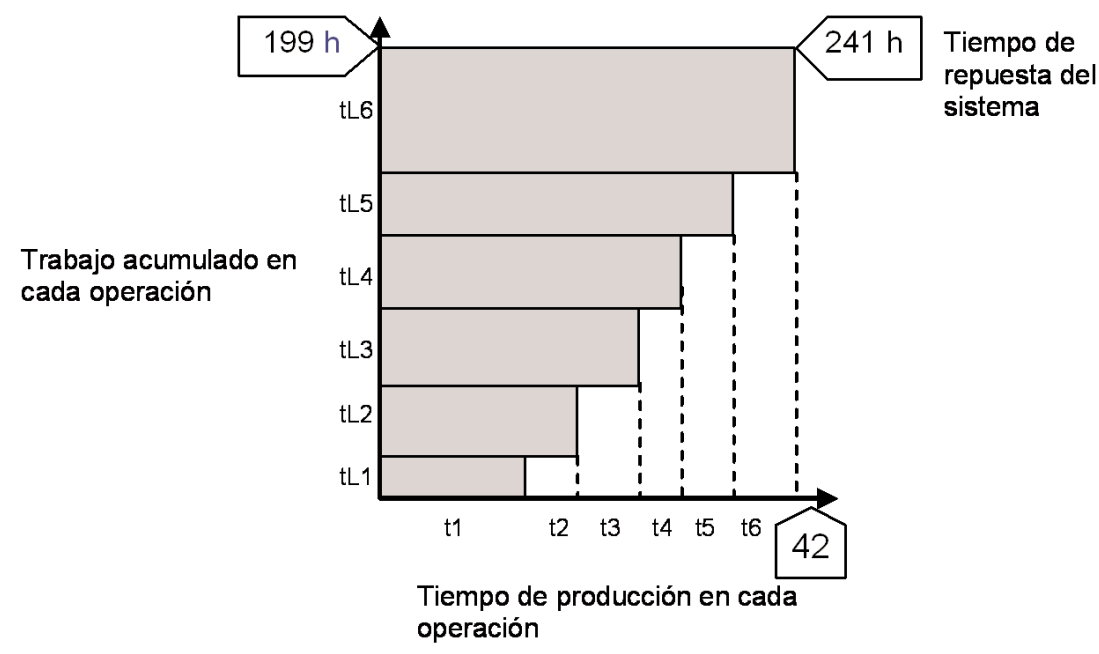




\subsection{Kaizen}

Esta filosofía de gestión significa mejoramiento continuo y se fundamenta en la implementación de sistemas que interactúan generando una importante sinergia. La estrategia Kaizen propicia el establecimiento de la gestión con calidad total (TQM), el sistema "justo a tiempo" (JIT), el mantenimiento productivo total (TPM), el despliegue de políticas y objetivos, el sistema de sugerencias y las actividades en pequeños grupos, para lograr la competitividad de las empresas.

Por definición, el Kaizen propicia el incremento del valor agregado evaluando el output de los procesos desde los enfoques de calidad, productividad o costos y oportunidad o servicio, tratando de mejorar los medios y métodos para eliminar, reducir o cambiar lo que sea necesario con el fin de evitar el despilfarro. La aplicación del Kaizen incluye la implantación de Wastology para obtener los cinco ceros (cero defectos, cero inventarios, cero paralizaciones, cero set-up-time, cero burocracia), lo que significa la eliminación del despilfarro; la aplicación de las cinco "S" para alcanzar la disciplina (seiri, seiton, seiso, seiketsu y shitsuke) e influir en la seguridad, calidad, eficiencia y eliminación de trastornos en la empresa; y la utilización de la estandarización de los procesos para conseguir la consistencia, indispensable para toda empresa.

\section{Planteamiento de acciones para la mejora empleando el método Kaizen Aplicación de las cinco " $S$ "}

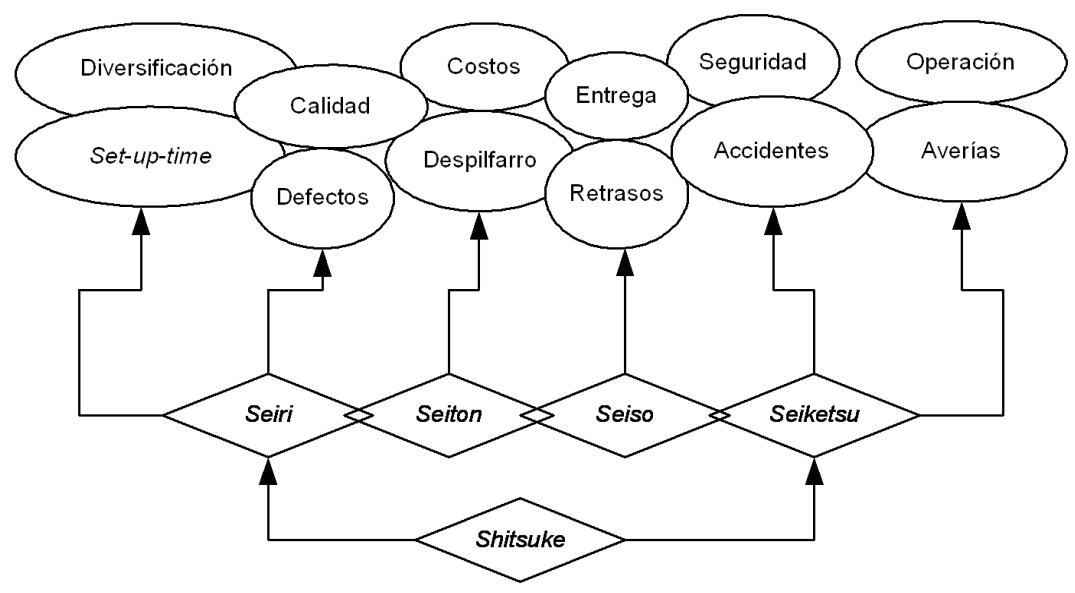


El Kaizen permite cambiar y mejorar la empresa, solucionando los problemas y subsanando las deficiencias, con el apoyo, participación y compromiso del personal de la empresa, que se responsabiliza de reconocer el problema, resolverlo e implantar la solución.

\section{Aplicación del Kaizen}

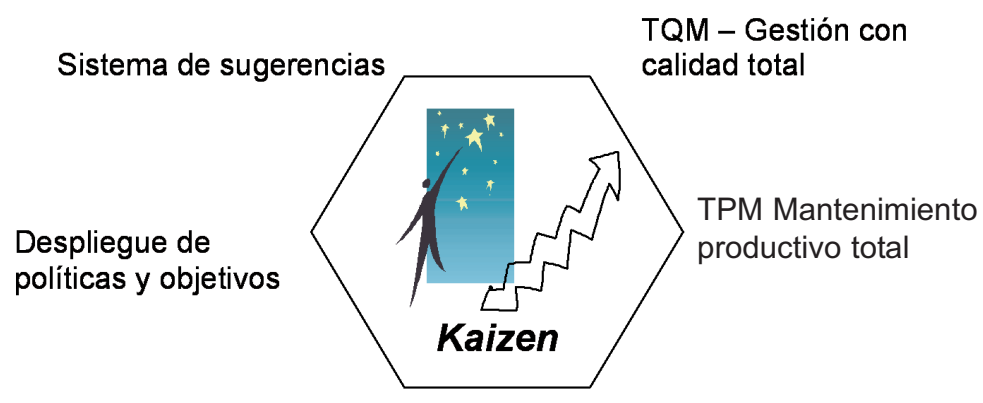

Trabajo en pequeños grupos

JIT - Justo a tiempo
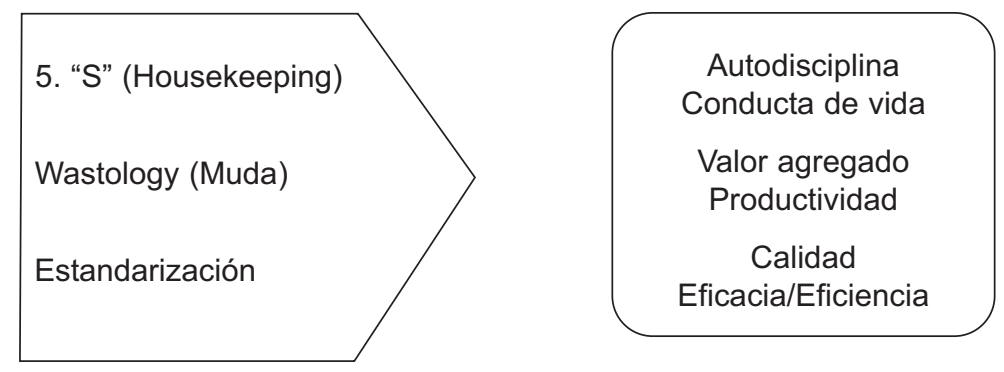

\section{IMPLANTACIÓN DEL MÉTODO EN EMPRESAS CASO}

El estudio está basado en la evidencia empírica de empresas ubicadas en la ciudad de Lima, donde se concentra el 30\% de la población peruana.

Una investigación empírica basada en los resultados obtenidos en una pequeña muestra dificulta la generalización de los resultados; sin embargo, se puede aceptar dicha generalización (Rantanen, Ukko y Rehhn), puesto que las empresas caso son percibidas como casos típicos, de acuerdo con las características definidas previamente.

La metodología diseñada se aplicó en varias pymes peruanas del sector industrial, la mayoría de ellas del sector metal mecánico. 
La empresa A es una pyme que produce vidrios y cuenta con dos locales: uno donde funcionan la administración y las ventas y el otro donde funciona la planta productiva, que está dividida en dos líneas de producción.

La empresa B es una pyme que fabrica pernos para la industria automotriz, cuenta con dos locales, uno donde funcionan las áreas de administración, ventas y producción, y otro donde funcionan una tienda y el almacén de productos terminados.

La empresa $\mathrm{C}$ es una pyme que fabrica máquinas de soldadura eléctrica y tiene un local con todas las facilidades, donde funciona la empresa en su totalidad.

La empresa D es una pyme que fabrica estructuras metálicas, tiene dos locales, uno donde funcionan la administración y las ventas y otro donde se encuentra la planta productiva.

El procedimiento de implantación del método consistió en la realización de reuniones de trabajo y workshops en cada una de las empresas, donde participaron representantes de estas y del equipo de investigación. En las reuniones se tomó contacto con el personal de la empresa, se recogió información y se concertó su participación en los workshops, se proporcionó el conocimiento teórico y práctico básico de las técnicas y herramientas, y se facilitó su aplicación en las empresas.

\section{RESULTADOS}

Los resultados obtenidos pueden ser enfocados desde dos puntos de vista. En primer lugar, en relación con la facilidad para el proceso enseñanza-aprendizaje de las técnicas y herramientas, lo que se logró con relativa facilidad, principalmente por su sencillez y facilidad de aplicación. En este caso, el método de mejoramiento fue utilizado sin ningún contratiempo por el equipo de trabajo conformado por personal de las empresas y por consultores externos.

En segundo lugar, en relación con los resultados obtenidos, es decir, de las mejoras alcanzadas en la práctica por las empresas. Al respecto, algunos de los resultados que se alcanzaron gracias a la aplicación del método de mejoramiento son los siguientes: 
- Disminución del inventario de materiales hasta un 51\% de la cantidad encontrada.

- Disminución del inventario de productos en proceso hasta un $60 \%$ de la cantidad original.

- Disminución de plazos de fabricación hasta en 29,5 días.

- Incremento de las piezas producidas por día hasta en un $205,5 \%$

- Incremento de los estándares en piezas por hora de hasta un $162,5 \%$.

- Mejora de la productividad en kg despachados/total de horas hombre de hasta $44,9 \%$.

- Productividad más consistente al mejorar su desviación estándar de 1,06 a 0,46 .

- Disminución del sobretiempo a solo el $22 \%$ de las horas extras semanales usuales.

- Implantación de una nueva organización estructural con puestos de trabajo bien definidos, con base en objetivos transparentes y tareas delimitadas.

- Asignación de carga de trabajo uniforme a los puestos de trabajo.

- Racionalización de la información.

\section{CONCLUSIONES}

Este trabajo describe el diseño y aplicación de un método de mejoramiento para las pymes, que fue desarrollado a partir de sus propias características y ha sido aplicado en varias pymes en el Perú con resultados beneficiosos para las empresas. De los resultados obtenidos en la aplicación de la metodología se puede inferir lo siguiente:

- Es muy importante que las técnicas y herramientas tengan un enfoque gráfico, puesto que esto ayuda a su entendimiento y facilita la interpretación de los resultados.

- El método es fácil de entender y su aplicación es sencilla.

- Los resultados obtenidos por las empresas peruanas en la práctica son buenos.

- El método puede aplicarse a las pymes de otros países en desarrollo sin necesidad de hacer ajustes o adecuaciones. 
Los autores creen que este trabajo debiera ser profundizado y que el método podría ser mejorado, hasta convertirse en un instrumento de apoyo para lograr el éxito de las pymes.

\section{BIBLIOGRAFÍA}

Barber, Kevin; Baron, John y Eric Gill. "A business improvement analysis to provide a methodology for the introduction of process improvement in SMEs based upon quality tools and procedures". ICPR 16. Praga, 2001.

Barcelli, Guillermo. "Herramientas analíticas para la consultoría". Separata. Diplomado en Consultoría en Comercio Internacional. Lima: Cámara de Comercio de Lima, 2003.

—. "Diagnóstico Empresarial". Curso. Lima: Universidad de Lima, 2002.

Del Carpio, Javier. "Perú país de pymes". Industrial Data 1. Lima, julio del 2000.

Dellaméa, Ricardo. Presencia 122. Lima: Confiep, 2001, pp. 38-39.

Duisberg Gesellschaft, Carl. CDG. "Programa de Administración de la Producción Industrial, Sistemas de información empresarial/Registro de datos de una empresa". Tema 14. Alemania, 1995.

El Comercio. "Pulso Latinoamericano". Lima, marzo del 2001.

García, Petronila. "Perú país de pymes". Industrial Data 1. Lima, julio del 2000.

Government Information Office Republic of China. "La pequeña y mediana empresa: Fuerza motriz de la economía de Taiwán". [en línea]. <http://www.gio.gov.tw/info/nation/sp/mse/smesp.htm>.

Greis, Peter. Globalisation - Blessing and burden, metallurgical plant and technology international. 5. Germany, 2001.

Hines, Peter y Nick Rich. "The seven value stream mapping tools". International Journal of Operations and Production Management. Vol 17, núm. 1, 1997, pp. 46-49.

Infante, Juan. Entrevista. El Comercio. Lima, 28 de diciembre del 2002. 
Masaaki, Imai. Cómo implementar el Kaizen en el sitio de trabajo (Gemba). Bogotá: McGraw-Hill Interamericana S.A., 1998.

Mendes, Luis y Luis Lourenço. "Quality management decisions in small business context: What about stakeholders?". ICPR 16. Praga, 2001.

Meyer-Stamer, Jorg. "Globalización y competencia. Las ventajas competitivas locales continúan siendo esenciales". Desarrollo y Cooperación $(D+C) 5$. Setiembre/octubre de 1997. [Versión original: Deutsche Stiftung fur internationale Entwicklung (DSE), C.C. 1206 23, D-53113 Bonn, Germany].

Niedereichholz, Christel. Gestión de la calidad en la consultoría empresarial. Lima: Confiep/GTZ, 1998.

OIT. La consultoría de empresas, 1997.

Pymes [en línea]. http://www.cajapymes.com/PyMEs.htm.

Rantanen, H; Ukko J.; Tenhunen, J. y M. Rehhn. "Implementation or a performance measurement system in SMEs in Finland". ICPR 16. Praga, 2001.

Sangmeister, Hartmut y Raquel Fuentes. "Competitividad: La batalla del desarrollo". Desarrollo y Cooperación $(D+C) 5$. Setiembre/octubre del 2002. [Versión original: Deutsche Stiftung fur internationale Entwicklung (DSE), C.C. 1206 23, D-53113 Bonn, Germany].

Valdivia, Néstor. Colaboración. El Comercio. Lima, 14 de febrero del 2002.

Wohlcke, Manfred. "El factor demográfico. Población, estabilidad política y medio ambiente en América Latina”. Desarrollo y Cooperación $(D+C)$ 2. Marzo/abril del 2001. [Versión original: Deutsche Stiftung fur internationale Entwicklung (DSE), C.C. 1206 23, D-53113 Bonn, Germany]. 